\title{
Coherence as a feature of real HF signals
}

\author{
James E. Giesbrecht ${ }^{a, b, c}$, Russell Clarke ${ }^{c}$, and Derek Abbott ${ }^{a, b}$ \\ ${ }^{a}$ Centre for Biomedical Engineering, The University of Adelaide, SA 5005, Australia \\ ${ }^{b}$ School of Electrical \& Electronic Engineering, The University of Adelaide, SA 5005, Australia \\ ${ }^{c}$ Ebor Computing, 147 Henley Beach Rd., Mile End, SA 5031, Australia
}

\begin{abstract}
High-frequency (HF) communications is undergoing a resurgence despite advances in long-range satellite communication systems. Defense agencies are using the HF spectrum for backup communications as well as for spectrum surveillance applications. Spectrum management organizations are monitoring the HF spectrum to control and enforce licensing. These activities usually require systems capable of determining the location of a source of transmissions, separating valid signals from interference and noise, and recognizing signal modulation. Our ultimate aim is to develop robust modulation recognition algorithms for real HF signals that propagate by multiple ionospheric modes.

One aspect of modulation recognition is the extraction of signal identifying features. The most common features for modulation recognition are instantaneous phase, amplitude, and frequency. Many papers present results based on synthetic data and unproven assumptions. However, this paper continues our previous work by applying the coherence function to noisy real HF groundwave signals; which removes the need for synthesized data and unrealistic assumptions.
\end{abstract}

Keywords: Modulation Recognition, Noise, Coherence, HF.

\section{INTRODUCTION}

The HF radio band (nominally $2 \mathrm{MHz}$ to $30 \mathrm{MHz}$ ) can be used for long-distance wireless communications, because the ionosphere and its various layers refract transmissions in this band. Such refraction enables signals to propagate beyond the horizon to distant receivers unable to be reached by higher frequency (VHF and above) signals. This fact makes the HF band attractive for private and commercial interests as well as for defense forces spread across the globe. For example, spectrum management agencies monitor the HF band for unlicensed operators and military agencies use the HF band for communications.

In the past, HF communication systems were analog. This meant that signals received by an antenna were down-converted to baseband using filters, oscillators, and many discrete components. Receivers were generally constructed in a super-heterodyne configuration. ${ }^{1}$ The baseband signals were then passed through demodulators to extract the information content. Often special demodulators had to be switched in to demodulate signals with different modulation schemes.

Today there are so many different modulation techniques that monitoring and detection of such signals using traditional methods would require numerous HF receivers. Monitoring and detection would also require some prior knowledge of the signals so as to choose the correct receiver. However, with the advent of software radio this task is much easier.

Software radio aims to replicate hardware functions in software running on a generic platform. In so doing many of the problems associated with hardware implementations are avoided. In addition, the receiver and transmitter chains can easily be changed to accommodate various modulation schemes. Here is where modulation recognition plays an important role. Modulation recognition enables a receiver to automatically switch in the correct software defined demodulator for a particular signal.

Send correspondence to:

The University of Adelaide - E-mail: james@eleceng.adelaide.edu.au; Phone: +61 (8) 83036296

Ebor Computing - E-mail: james.giesbrecht@ebor.com; Phone: +61 (8) 8238 0300; Fax: +61 (8) 82380304 
In the previous work ${ }^{2-4}$ we discussed the fundamentals of coherence and entropy as well as the preliminary application of coherence ${ }^{5}$ and entropy ${ }^{6}$ to real HF signals. This work looks more closely at the feature of coherence for real HF signals and how it might be used in modulation recognition.

\section{MODULATION RECOGNITION}

The process of determining the modulation type of a signal with no foreknowledge of the signal modulation characteristics is known as modulation recognition. Fundamental processes of modulation recognition are feature extraction and classification. Feature extraction determines unique characteristics of the signal so that a classifier can establish the modulation type. Common features include instantaneous amplitude, variance of phase, spectral symmetry, transmission models, and higher order statistics. Classification associates features with modulation types. These associations are normally made through threshold detection logic, artificial neural networks (ANNs), or pattern recognition algorithms.

Previous work ${ }^{2-4}$ on real HF signals has shown that most recognition methods do not adequately rationalize the choice of thresholds and feature functions. Moreover, they do not assume appropriate noise and channel models for application of their methods to the HF band, and they do not apply their methods to real signals. Figure 1 illustrates power spectra of typical HF signals.

Nevertheless, the most common features are statistical measures of instantaneous amplitude, phase, and frequency. ${ }^{7-10}$ Others have considered zero-crossings, covariance, and higher-order statistics (e.g. $\mathrm{m}^{\text {th }}$-order cumulants and kurtosis). ${ }^{11-15}$ This work concentrates on the feature of coherence.
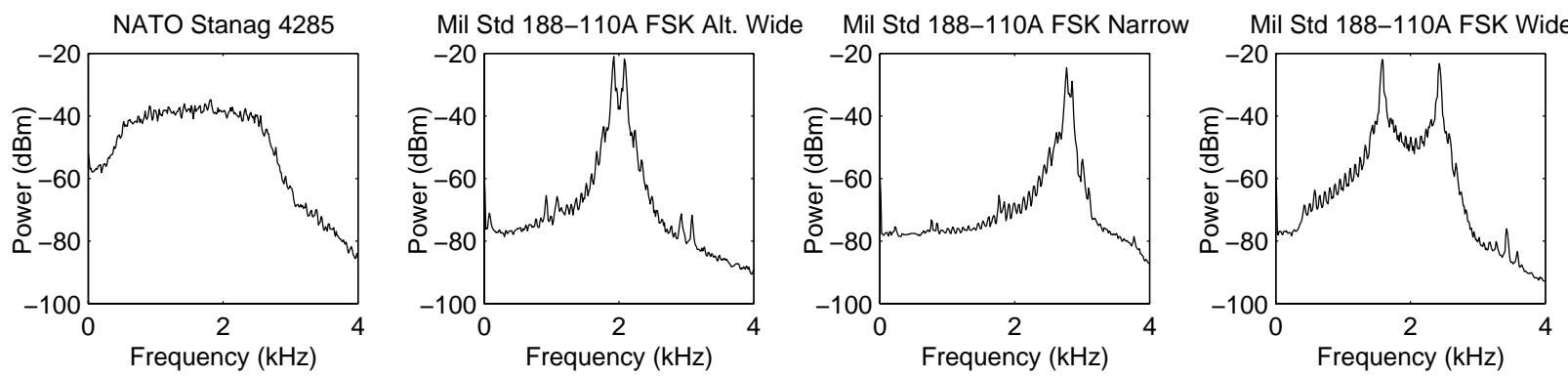

Figure 1. Power spectra of various real HF signals. The NATO Stanag 4285 is an 8-PSK signal while the other plots are of 2-FSK signals.

\section{COHERENCE AS A SIGNAL FEATURE}

The coherence function is analogous to correlation coefficients in the frequency domain. Consider the powerspectral density of a signal $X$. It is defined as

$$
P_{x x}(f)=\int_{-\infty}^{\infty} \rho_{x x}(\tau) e^{-j 2 \pi f \tau} d \tau
$$

where $f$ is frequency, $\tau$ is time delay, and $\rho_{x x}(\tau)$ is the auto-correlation function of $X$. In a similar manner, the cross-spectral density is defined by

$$
P_{x y}(f)=\int_{-\infty}^{\infty} \rho_{x y}(\tau) e^{-j 2 \pi f \tau} d \tau
$$

where $\rho_{x y}(\tau)$ is the cross-correlation of signals $X$ and $Y$. From these definitions the coherence function is the square of the cross-spectral density normalized by the respective power-spectral densities;

$$
C_{x y}(f)=\frac{\left|P_{x y}(f)\right|^{2}}{P_{x x}(f) P_{y y}(f)} .
$$


For each frequency, the coherence function varies between 0 and 1 and indicates the similarity between $X$ and $Y$. If the value of the function at a particular frequency is close to unity, it indicates that $X$ and $Y$ are similar at that frequency. On the other hand, if the value of the function is near zero it implies that the two signals are dissimilar at the particular frequency. Furthermore, if the coherence is unity it necessarily means that $X$ and $Y$ are correlated.

Carter's ${ }^{5}$ excellent treatise on the estimation of coherence shows that the coherence between $X$ and $Y$ is also related to the signal-to-noise ratio (SNR) of $Y$ assuming that $X$ is the transmitted signal and $Y$ is the noisy received signal. In this description

$$
C_{x y}(f)=\frac{\operatorname{SNR}_{y}(f)}{1+\operatorname{SNR}_{y}(f)},
$$

where

$$
\operatorname{SNR}_{y}(f)=\frac{P_{x x}(f)|H(f)|^{2}}{P_{n n}(f)},
$$

and where $H(f)$ is the transfer function of the transmission channel and $P_{n n}(f)$ is the power-spectral density of the received noise. Note that with this definition, a coherence of unity not only demonstrates complete correlation of $X$ and $Y$ but also implies an infinite SNR since in the limit as $\operatorname{SNR}_{y}(f) \rightarrow \infty$ the coherence tends to 1 .

As shown earlier, ${ }^{4}$ despite the simplistic appearance of (3) and (4) the calculation and measurement of coherence can be quite difficult. For example Proakis ${ }^{16}$ demonstrates that the calculation of the power spectral densities of FSK signals, and therefore coherence, is not an easy task. Therefore coherence is usually estimated. Carter $^{5}$ points out that the coherence function between two signals is extremely sensitive to misalignment in time*. He suggests that the best methods to estimate coherence are weighted overlapping segment averaging (WOSA) (e.g. Welch's periodgram method ${ }^{17}$ ) and lag reshaping. For each of these techniques the signals are broken into windowed overlapping segments. As the number of segments increase, the variance of the coherence estimate decreases. Moreover, the percentage of overlap between segments affects the bias. The bias in the estimate of coherence decreases as the percentage overlap increases. However there is a point of diminishing returns (i.e. about 50\% - 60\% overlap) where further increase in overlap provides minimal reduction in the bias. Consequently, an accurate estimate of coherence depends on the choice of number of segments, percentage overlap, and windowing method.

So, is coherence a useful feature for modulation recognition? If so, how can coherence be used to identify a signal's modulation?

\section{RESEARCH PLATFORM}

An HF Monitoring System and research platform is currently under development at Ebor Computing. This monitoring system consists of an array of antennas, followed by signal conditioning electronics, multiple digital receivers, and a data processing sub-system (see Figure 2).

The Analog RF Subsystem consists of antennas, amplifiers, attenuators, and filters. Outputs of this subsystem are fed to a rack of digital receivers that directly sample RF signals up to $50 \mathrm{MHz}$ and down-convert the $\mathrm{HF}$ channels to baseband. The digitization of the RF signals is achieved by high-speed analog-to-digital converters (ADCs). Down-conversion is accomplished with digital-down-converters (DDCs). The Data Processing Subsystem collects the baseband information and processes it. Processing includes functions such as modulation recognition, signal separation, and SSL. A Remote Management System oversees the operation of the entire system. The research platform operates independently of the Data Processing Subsystem and Remote Management System. It also includes a remotely-controlled test-transmitter with HF modem (not shown) that sends groundwave signals to the HF Monitoring System. The research platform (see Figure 3) consists of two main components: feature extraction and modulation recognition. The feature extraction component determines features, such as coherence, from real or synthetic signals. The modulation recognition block uses the extracted features to classify the received baseband signal.

\footnotetext{
${ }^{*}$ We shall see in Section 6 that misalignment can result in a very low coherence.
} 


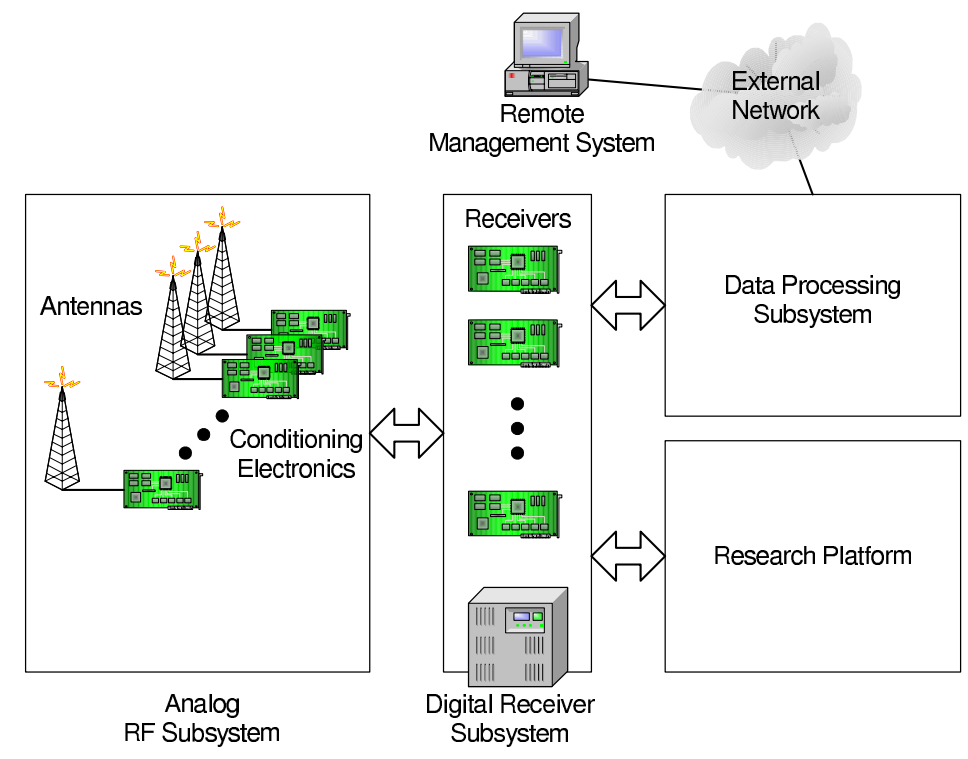

Figure 2. Architecture of the HF monitoring system.

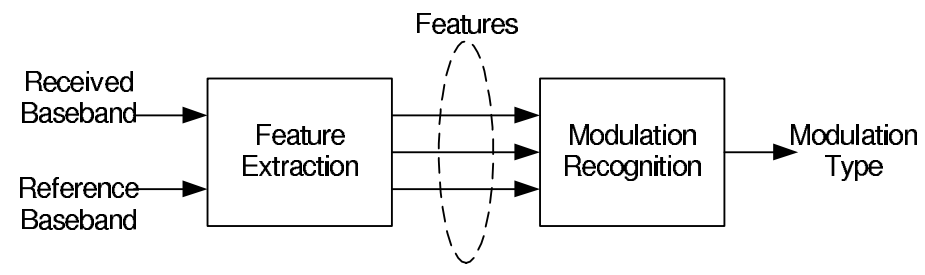

Figure 3. Fundamental components of research platform. The feature extraction component is implemented while the modulation recognition block is a topic of future work.

\section{DATA COLLECTION}

An HF modem (GEC-Marconi ARM-9401) was configured to output a baseband signal (-40 dBm modulation level) into an $\mathrm{HF}$ transmitter (1 W transmission at $15.824 \mathrm{MHz}$ ) a few kilometers from the antenna array of the HF Monitoring System. At this distance, the HF Monitoring System was able to receive groundwaves from the transmitter that arrived relatively unattenuated and undistorted but still containing noise and interfering signals. The modem was capable of a number of different modulation schemes (some are listed in Table 1), however, only FSK Alt. Wide (hereafter referred to as FAW) was considered in this work.

\section{METHOD AND RESULTS}

The research platform generated an BFSK reference signal with the same mark and space frequencies as the FAW signal. Both signals carried messages consisting of random bits, but the FAW signal was a framed signal whereas the reference signal was not framed.

As can be seen in Figures 4 and 5, the power-spectral-densities of the reference signal and the real signal are very different and therefore it is no surprise that the coherence in Figures 6 and 7 show no significant peaks at the mark and space frequencies. It so happens that Trial 33 (or thereabouts) in Figure 6 yields the highest coherence. Clearly in this trial the synthesized FSK signal more closely matches the real signal than do the other trials. And, even though there is a wall of peaks about $2 \mathrm{kHz}$, the generally low coherence is less than ideal. We 
Table 1. Some modulation types of the ARM-9401 HF modem.

\begin{tabular}{lll}
\hline \hline Modulation & Standard & Characteristics \\
\hline \hline 8-PSK & Stanag 4285 & 75 baud long interleaving \\
& & $\begin{array}{l}\text { Single Tone Sub-Carrier } 1800 \mathrm{~Hz} \\
\text { Bandwidth 600-3000 Hz }\end{array}$ \\
\hline FSK Alt. Wide & Mil-Std-188-110A & 75 baud no interleaving \\
& Sec. 5.1 .1 (HF Narrow) & Mark:1915 Hz Space:2085 Hz \\
\hline FSK Narrow & Mil-Std-188-110A & 75 baud no interleaving \\
& Sec. 5.1 .3 (LF UHF Speech+Telephony) & Mark:2762.5 Hz Space:2847.5 Hz \\
\hline FSK Wide & Mil-Std-188-110A & 75 baud no interleaving \\
& Sec. $5.1 .2(\mathrm{HF}$ Wide) & Mark:1575 Hz Space:2425 Hz \\
\hline
\end{tabular}

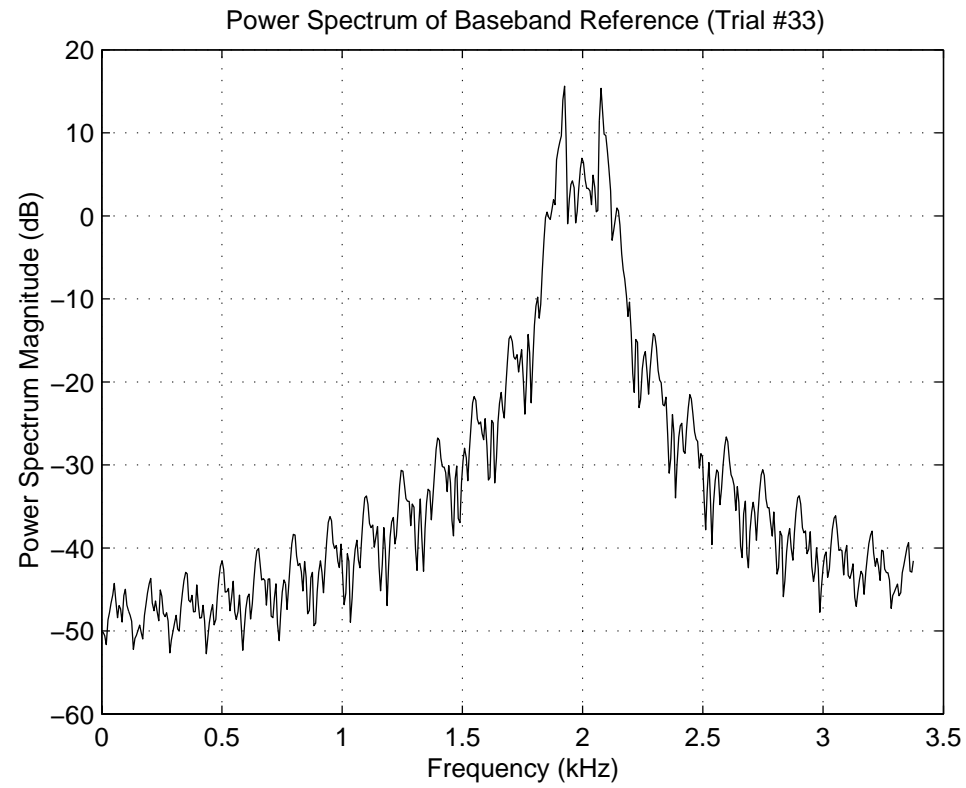

Figure 4. A synthetic BFSK signal used as a baseband reference. The message carried by the signal consists of random bits. The peakedness in the spectrum is an artifact of the random message.

desire the coherence to be significantly higher (i.e. much closer to unity) than the surroundings in order to be definitive about the classification of a signal based on the coherence.

Consequently, this experiment verifies statements made earlier, ${ }^{4}$ that the coherence will only yield a yes/no answer as to whether or not the received signal is the same as the reference signal. If the reference signal is changed to an ideal FAW signal, then perhaps the coherence would be greater. This however does raise the question that perhaps the coherence would be high if a receiver were to maintain a local copy of a such a signal instead of the synthetically generated BFSK.

So, what if the signal reference is a real FAW signal instead of synthesized BFSK? Here we use the signal in Figure 5 as the baseband reference signal and the signal in Figure 8 as the noisy received signal. Both signals are FAW signals acquired at different times on different antennas of the same antenna array. The power spectra of the two signals are strikingly similar and consequently one would expect the coherence to be high. But, contrary to what we might naively expect, the coherence in Figure 9 is very low.

We see that near the mark and space frequencies (1915 Hz and $2085 \mathrm{~Hz}$ respectively) the coherence is lower 


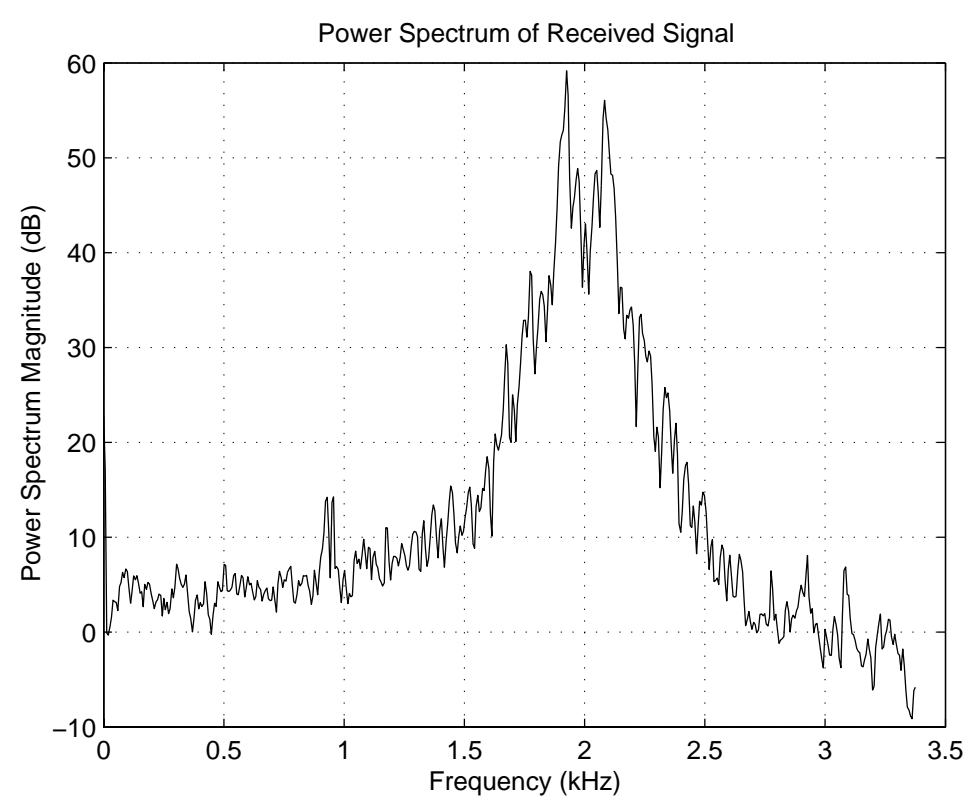

Figure 5. This is a real FAW groundwave signal captured by the research platform. Note the mixer products at about $1 \mathrm{kHz}$ and $3 \mathrm{kHz}$.

than in Figure 7! However, careful analysis of Figures 6 and 7 shows that in general the synthesized reference provides no better a result. We know from previous work ${ }^{4}$ that despite the similarities of the spectra, the message plays an important role in the coherence. The closer the messages of the two signals the larger the coherence. But, since the HF modem generated different random messages at different times we see that the resulting coherence is low.

Now we consider the case where the signal reference and the received signals are captured at the same time but on different antennas (see Figures 5 and 10). Because the signals carry the same message and timing the coherence in Figure 11 is unity in the passband of the signal. The fact that the coherence rolls off to something much less than one at frequencies outside the passband is a result of time delay (phase differences) between the signals received by the two antennas, and the various noise signals captured by each antenna that are independent of the other.

In the first experiment we used a BFSK signal against a FAW signal in the coherence calculation, which resulted in a low coherence. One would expect that comparing two FAW signals would produce a better coherence result. However as shown above, this is not necessarily the case. As in the first experiment, we would have to generate a number of trials with a number of FAW references and hope that one of the trials would yield a high coherence. This trial-and-error method is processing intensive, especially when trying to try to answer the question: how many trials are needed before we can be satisfied with the coherence results? For real-time software radio systems such a method is unsatisfactory. At best, using coherence to identify an unknown signal is a brute-force method suitable only for "non-real-time" analysis. Consequently, the use of coherence to identify an unknown real signal is not practical.

The preceding analysis dealt only with FSK type signals. One may argue that the results of the analysis may improve if a different modulation were chosen. Indeed, that may be the case. Yet, one of our goals is to develop a robust modulation recognition method that, though unlikely, recognizes a large portion of the most common modulation types. For HF communications, m-ary FSK related modulations are extremely popular. Therefore, if coherence cannot be successfully used to recognize even the most basic of modulation types it is of little use. 


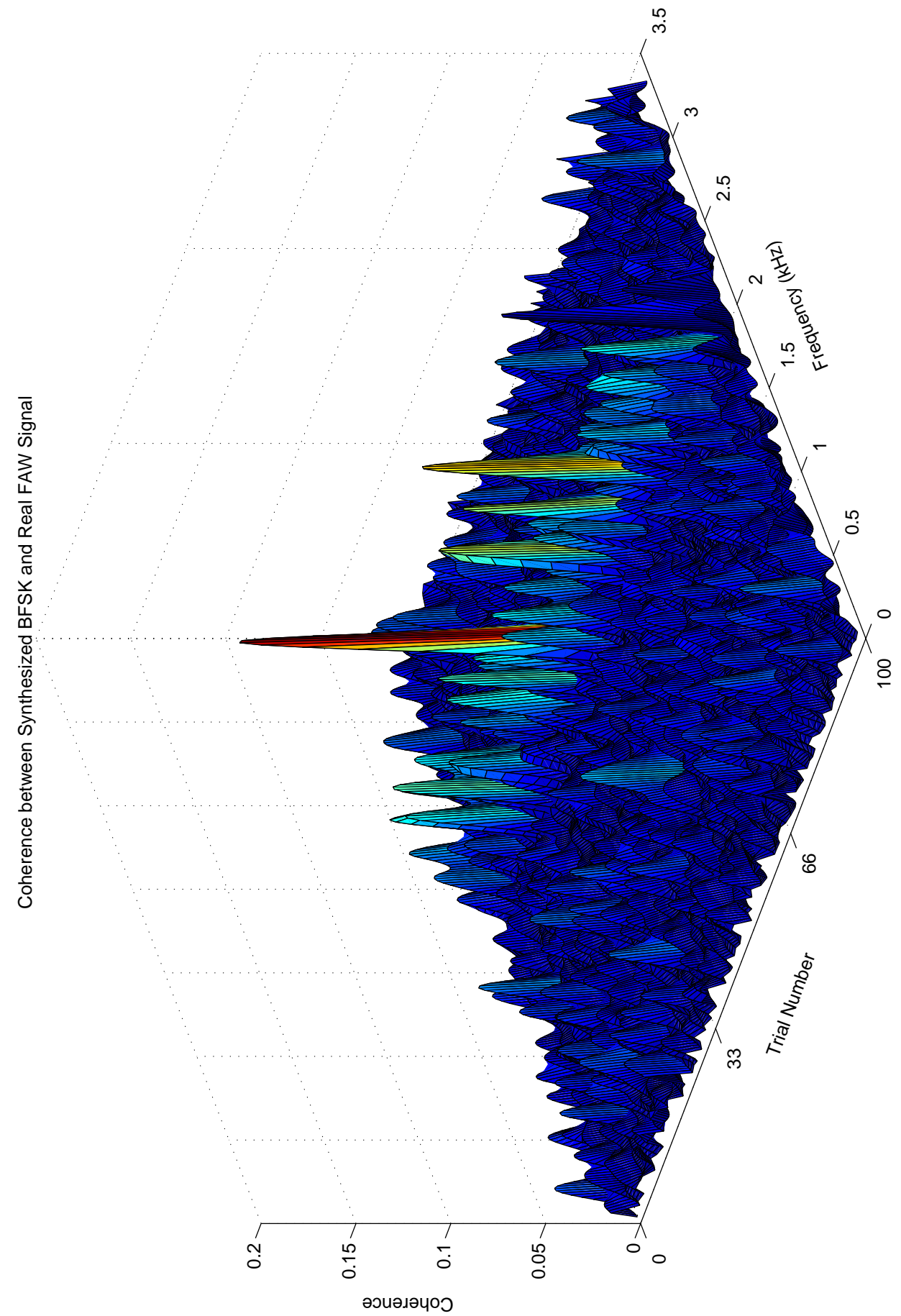

Figure 6. Coherence between synthetic FSK and real FAW signal. The maximum coherence occurs about Trial 33 . Note the valleys between the peaks along the wall at $2 \mathrm{kHz}$. For these trials the messages in the synthetic FSK signal do not match the message in the real FAW signal particularly well. The trials yielding peaks indicate a better matching of the messages. 


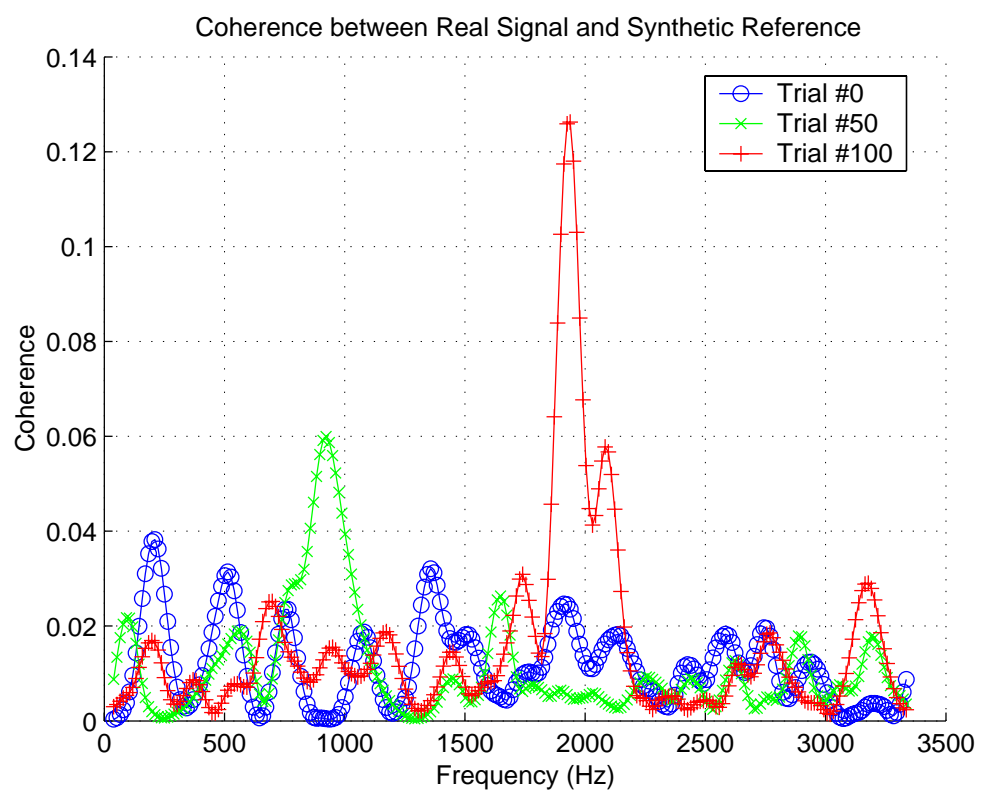

Figure 7. Coherence between the real FAW signal and the synthetic BFSK reference for various trials. Trial 100 appears to match the real signal at the mark and space frequencies (1915 Hz and $2085 \mathrm{~Hz}$ respectively) more closely than Trials 0 and 50. Compare these results with Figure 6.

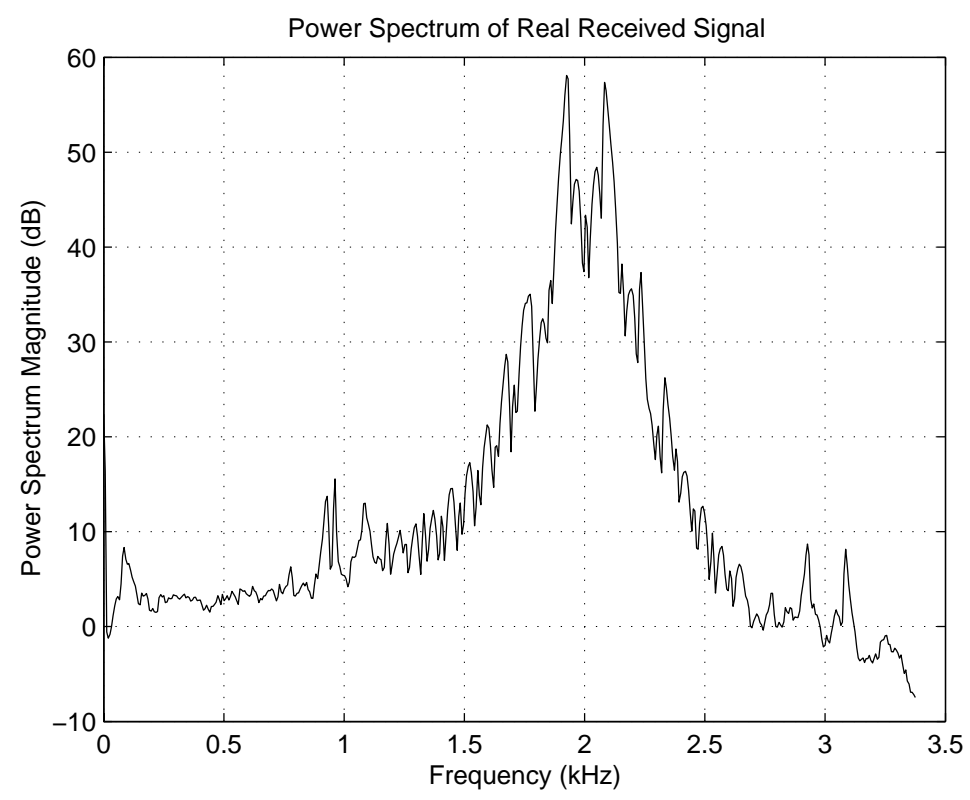

Figure 8. Power spectrum of a FAW signal acquired at a time and on an antenna different from the signal in Figure 5. The message content of each signal is different even though the spectra of the two signals are nearly the same. 


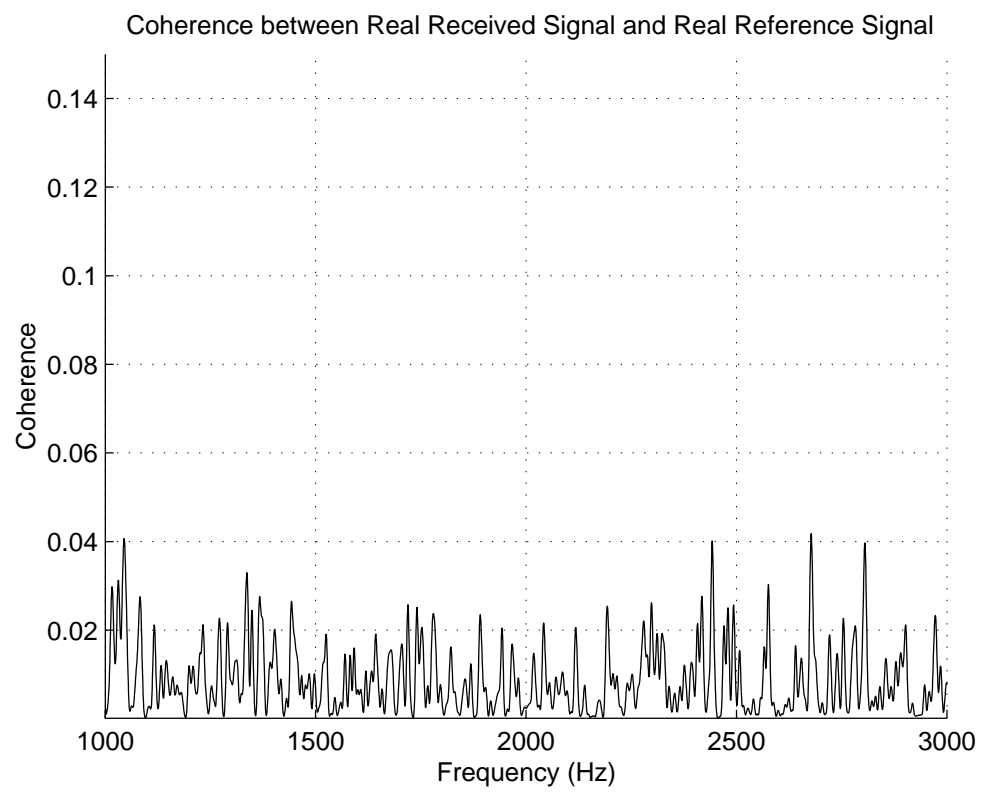

Figure 9. The coherence between the signals of Figures 5 and 8 is very low at the mark and space frequencies. The low coherence is primarily a result of the different messages carried by each signal. Other factors contributing to the low coherence is independent noise and interference.

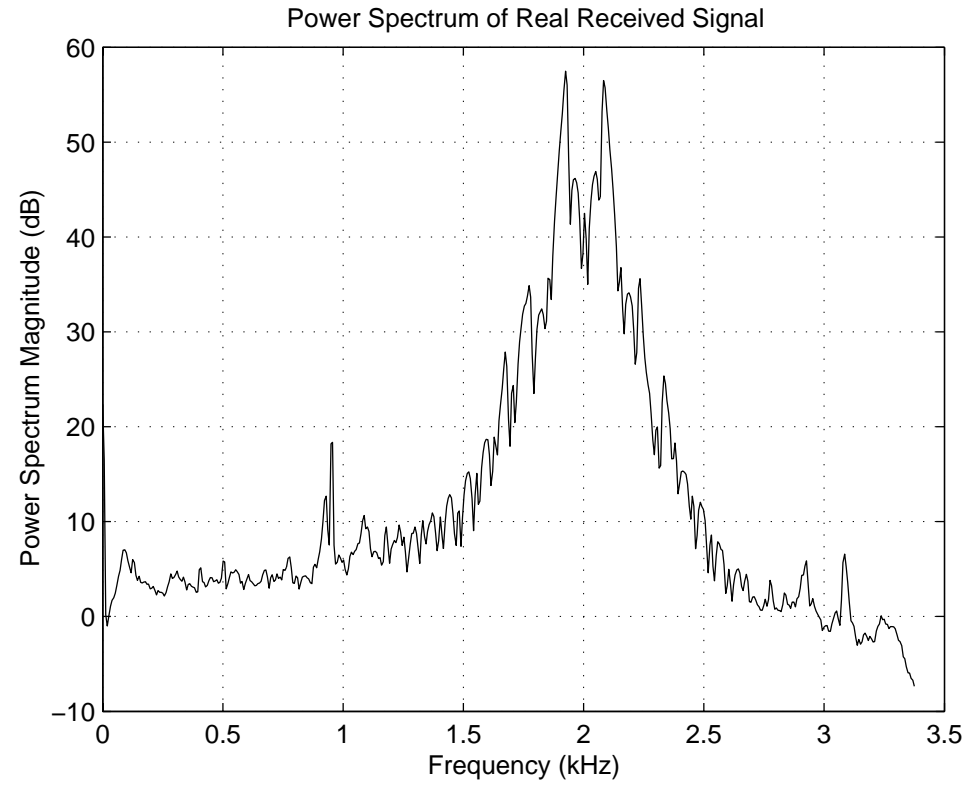

Figure 10. Power spectrum of a FAW signal received on antenna different from the antenna used to acquire the signal in Figure 5. Despite the striking similarities, careful inspection of the two signals reveals slight differences. These differences are due to time delay (hence phase difference) between the two signals as well as noise and interference from common and independent sources. 


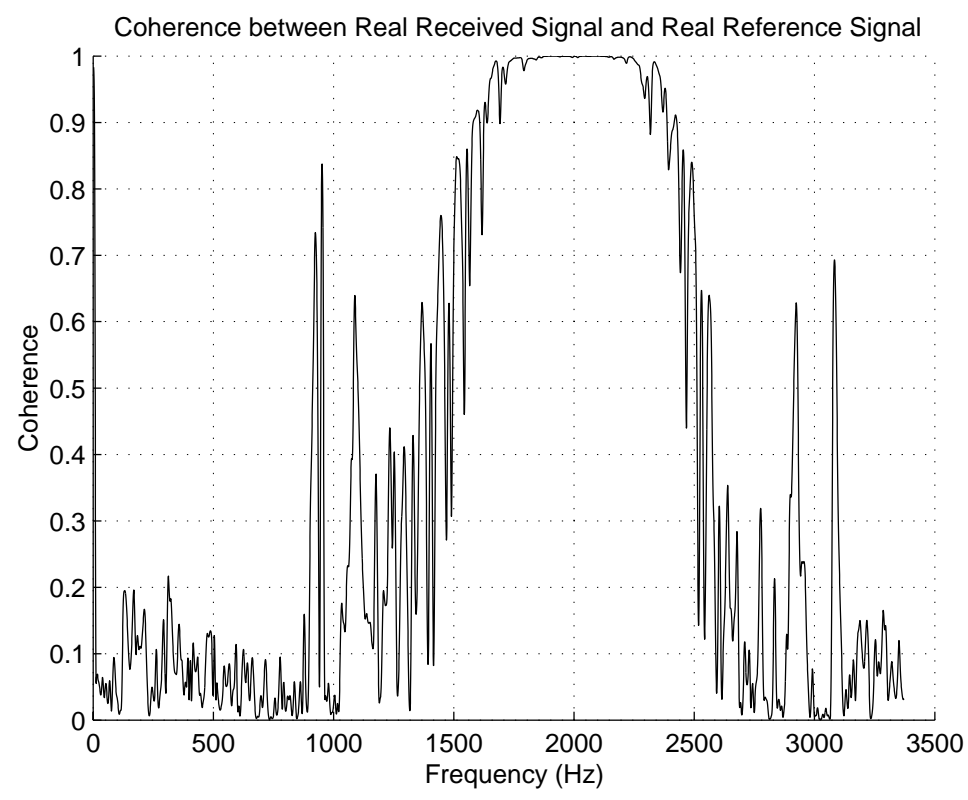

Figure 11. Coherence of signals (see Figures 5 and 10) received on two separate antennas but at the same time. Note the unity coherence in the passband (approx. $1900 \mathrm{~Hz}$ to $2100 \mathrm{~Hz}$ ). Outside the passband the coherence drops rapidly to near zero. The low coherence outside the passband is due to the independent components of the two signals at these frequencies.

\section{SUMMARY AND FUTURE WORK}

Three experiments are conducted that demonstrate the performance of the coherence function with real $\mathrm{HF}$ signals. The first test calculates the coherence between a synthesized BFSK reference and a real Mil-Std-188110A FSK signal. The resulting coherence is very low. The second experiment compares two real Mil-Std-188110A FSK signals acquired at different times and finds that the coherence is also very low. The low coherence is attributed to the difference in the messages carried by the signals. This was shown in previous work with simulated data ${ }^{4}$ and is again verified by the coherence between two real Mil-Std-188-110A FSK signals having the same message. In this case the coherence is unity in the passband and near zero outside the passband.

Consequently, the use of coherence to identify an unknown real signal is not practical. All that can be done is to perform a number of trials in which the reference signal maintains its modulation type, but has varying messages, in order to get some level of confidence in our guess as to the modulation type of an unknown received signal.

Future work will apply various other features described previously ${ }^{3,4}$ to sample sets of real HF groundwave signals. The aim is to find a useful set of features and to then apply them to HF skywave signals that are affected by phenomena such as multi-path, fading, and doppler shifts before ultimately developing a robust modulation recognition algorithm.

\section{ACKNOWLEDGMENTS}

Financial support for this work is provided by the The University of Adelaide and Ebor Computing. 


\section{REFERENCES}

1. J. Smith, Modern Communication Circuits, McGraw-Hill Series in Electrical Engineering, McGraw-Hill Inc., New York, 1st ed., 1986. pp. 1-13.

2. J. E. Giesbrecht, R. Clarke, and D. Abbott, "Improved techniques for monitoring the HF spectrum," in Proceedings of SPIE, 5274, pp. 112-122, 2003.

3. J. E. Giesbrecht, R. Clarke, and D. Abbott, "Monitoring the HF spectrum in the presence of noise," in Proceedings of SPIE, 5473, pp. 76-84, 2004.

4. J. E. Giesbrecht, R. Clarke, and D. Abbott, "Modulation recognition for HF signals," in Proceedings of SPIE, 5649, 2004. (In Press).

5. G. C. Carter, Coherence and Time Delay Estimation: An Applied Tutorial for Research, Development, Test, and Evaluation Engineers, IEEE Press, Piscataway, NJ, 1993.

6. D. Benedetto, E. Caglioti, and V. Loreto, "Language trees and zipping," Physical Review Letters 88(4), 2002. 048702 .

7. A. O. Hero III and H. Hadinejad-Mahram, "Digital modulation classification using power moment matrices," in Proceedings of the IEEE 1998 International Conference on Acoustics, Speech, and Signal Processing, 6 , pp. 3285-3288, (Seattle), 1998.

8. H. Ketterer, F. Jondral, and A. H. Costa, "Classification of modulation models using time-frequency methods," in Proceedings of the IEEE 1999 International Conference on Acoustics, Speech, and Signal Processing, 5, pp. 2471-2474, (Phoenix, Arizona), 1999.

9. M. L. D. Wong and A. K. Nandi, "Automatic digital modulation recognition using spectral and statistical features with multi-layer perceptrons," in Proceedings of the IEEE International Symposium on Signal Processing and its Applications, 2, pp. 390-393, (Kuala Lumpur, Malaysia), 2001.

10. K. E. Nolan, L. Doyle, P. Mackenzie, and D. O'Mahony, "Modulation scheme classification for 4G software radio wireless networks," in Proceedings of the IASTED International Conference on Signal Processing, Pattern Recognition, and Applications (SPPRA 2002), pp. 25-31, (Crete, Greece), 2002.

11. J. Aisbett, "Automatic Modulation Recognition Using Time Domain Parameters," Tech. Rep. ERL-0367TR, Electronic Research Laboratory DSTO, Department of Defence, Commonwealth Government of Australia, November 1986.

12. S.-Z. Hsue and S. S. Soliman, "Automatic modulation classification using zero crossing," IEE Proceedings 137 Pt. F, pp. 459-464, December 1990.

13. A. K. Nandi and E. E. Azzouz, "Modulation recognition using artificial neural networks," Signal Processing 56, pp. 165-175, 1997.

14. A. K. Nandi and E. E. Azzouz, "Algorithms for Automatic Modulation Recognition of Communication Signals," IEEE Transactions on Communications 46, pp. 431-435, April 1998.

15. W. Akmouche, "Detection of multicarrier modulations using 4th-order cumulants," in MILCOM 1999 Military Communications Conference, 1, pp. 432-436, Institution of Electrical and Electronic Engineers, October 1999.

16. J. G. Proakis, Digital Communications, Communications and Signal Processing, McGraw-Hill Book Company, 2nd ed., 1989.

17. P. D. Welch, "The use of the fast fourier transform for the estimation of power spectra: A method based on time averaging over short, modified periodograms," IEEE Transactions on Audio and Electroacoustics AU-15, pp. 70-73, June 1967. 